\title{
Insulin Resistance Syndrome- the sensitive IRS
}

2

3

Eleni Pellazgu, PhDc, MSN, APN

Rutgers University Graduate School of Nursing, NJ

Atlantic Health System, Morristown, NJ

Correspondence: pellazel@sn.rutgers.edu

Potential conflict of interest: Author declares no conflict of interest

Acknowledgement: Many thanks to the Department of Metabolic Medicine at Atlantic Health System for their mentoring and support all these years.

\section{Abstract}

Insulin resistance is a prominent pathophysiologic syndrome in a plethora of metabolic disorders including obesity, prediabetes, type 2 Diabetes Mellitus (Ten et al., 2007), impaired glucose tolerance, dyslipidemia, polycystic ovary syndrome and hypercoagulability (Smith \& LeRoith, 2004). It is strongly associated with obstructive sleep apnea, hypoventilation syndrome, pancreatitis, nonalcoholic fatty liver disease, steatohepatitis, cirrhosis, gall bladder disease, multiple cancers (breasts, uterus, cervix prostate, kidney, colon, esophagus, pancreas and liver), stroke, cataracts, coronary heart disease, and hypertension. It is also associated with causation of abnormal menses, infertility, polycystic ovary syndrome, osteoarthritis, phlebitis and other venous diseases.

Insulin resistance is thought to be caused by intrinsic and extrinsic factors that contribute to its development. Once present, insulin resistance affects the metabolism, behavior, physical 
appearance and has lasting effects. This paper will review the latest evidence in development of insulin resistance, its pathogenesis and manifestation and its relation to other conditions. The final aim is to raise awareness of its role on diet, metabolic, genetics and microbiome.

Keywords: insulin resistance, diet, exercise, microbiome, metabolic disorders

\section{Insulin resistance pathogenesis}

The term insulin resistance implies excess production of insulin as a response to triggering factors which makes the receptors overworked and overused to it therefore more insulin is needed to achieve needed effect. Insulin resistance was first described in 1936 to illustrate a metabolic disturbance showing impaired cellular responsiveness to insulin signaling in insulin dependent tissues such as skeletal muscle, liver, and adipose tissue (Bermudez et al., 2016). Studies point to alarming projections for the future with an estimated prevalence of $33 \%$ of the adults in USA alone (Bermudez et al., 2016). Insulin resistance syndrome (IRS) has a domino effect mostly associated with the liver, muscle, adipose tissue and endothelium. Insulin causes a powerful shift of the metabolism towards hepatic triglyceride production, cholesterol synthesis, followed by transport and tissue accumulation of the lipids produced. However, the continued flow of insulin production at the level of the pancreatic beta cell leads to eventual exhaustion of the insulin production and secretion apparatus, eventually causing type 2 diabetes mellitus (T2DM). The ongoing hyperinsulinemia leads to significant dyslipidemia and vascular inflammation, producing cardiovascular disease (Lopez-Candales, Hernández Burgos, Hernandez-Suarez, \& Harris, 2017).

The deposition of excess glucose and end products glucose of metabolism in sensitive tissues such as the brain, is believed to have a causative role in the development of Alzheimer's disease, (also classified as a type of diabetes) (de la Monte \& Wands, 2008). Insulin inhibits 
gluconeogenesis and promotes glycogen production and once the glycogen stores are maximized, insulin stimulates the conversion of glucose into fatty acids. When carbohydrates are abundantly present, the body enters in a process of producing more and more insulin to regulate the glucose metabolism which may cause drop in blood glucose and ingestion of more carbohydrates to counteract effects of reactive hypoglycemia therefore starting a vicious cycle. This is because of the metabolic consequences of insulin excess in the presence of high blood sugar levels.

Intrinsic behaviors such as compulsive eating and stress eating have been associated with insulin resistance. Overeating is one of the major causative factors that induces low grade inflammation secondary to activation of various transcriptional mediated molecular a metabolic pathways which happen due to elevated glucose and lipids in blood stream (Paniagua, 2016). Other intrinsic factors include metabolism, genetics, microbiomes behavior and pathologic conditions. The extrinsic pathogenic factors for insulin resistance include diet, physical activity, social pressures, toxins and medications. In the latter category, meds such as statins, thiazides, beta blockers, antiretroviral, steroids, antiestrogens, chemotherapy and antirejection drugs are known to affect glucose metabolism (Bays, 2011). Once, IRS is developed, it increases the oxidative stress in $b$ cells of pancreatic islets and peripheral tissues. To counter these processes, the best approach is to prevent the pathogenies of IRS thru ameliorating the incidences of inflammatory responses (Rehman \& Hamid Akash, 2016).

\section{Metabolic syndrome}

Metabolic syndrome (MetS) is considered a IRS surrogate (Singh et al., 2012) and represents a clinical diagnostic entity identifying the individuals at risk with cardiovascular morbidity linked to IR (Wilcox, 2005). MetS is a cluster of five condition with three of them confirming diagnosis. The five criteria for diagnosis are abdominal obesity, 
hypertriglyceridemia, low HDL-C, hypertension and elevated fasting glucose. Regardless of various names and definitions, it is estimated that nearly 100 million Americans or about $30.5 \%$ have MetS, mostly undiagnosed. IRS is suggested to be a major factor linking physical inactivity and MetS (Roberts, Hevener, \& Barnard, 2013). Together, increased consumption of refined sugars, saturated fats, and increased calorie intake, linearly correlate with increased prevalence of obesity, diabetes and other components of metabolic syndrome (Siegfried, Shiho, \& Kahn, 2016).

\section{Genetics and insulin resistance}

The most common genetic obesity condition resistant to weight loss are the Prader Willi syndrome occurring in one out of 20,000 births followed by rarer conditions such as the Bardet Biedl syndrome, the Cohen and Ayazi syndrome (Gunay-Aygun, Cassidy, \& Nicholls, 1997). The recently discovered, non-syndromic genetic obesity patterns have the opposite characteristics. Variations in ADIPOQ are associated with obesity, type 2 diabetes (T2DM) and related phenotypes in several populations including Asian populations (Siitonen et al., 2011). They result in a reduction in adiponectin, an adipocyte hormone that reduces inflammation, dyslipidemia and insulin resistance. On the other hand, liver also has an important role in nutrition metabolism thru regulation via production of the hepatokine fibroblast growth factor 21 (FGF21), which significantly suppresses consumption of simple sugars, but not complex carbohydrates, proteins, or lipids. Von Holstein et al, 2016 believe that the liver regulates the intake of certain micronutrients by increasing endocrine satiety meaning that literally suppresses the intake of sugars since its elevated numbers positively correlate with BMI, waist circumference (WC), fat mass and central adiposity (Chu et al., 2013; von Holstein-Rathlou et al., 2016). Chu et al, 2013 performed a large study of a genome-wide meta-analysis of 33355 
male and female participants of European ancestry in DietGen consortium consisting of three large population-based studies, investigating the genetics of dietary intake and nutrition and found results that demonstrate associations with macronutrient intake at genome-wide significance (Chu et al., 2013; Heianza et al., 2016).

\section{Microbiome contribution}

This is an emerging field and we are now recognizing the role that the bacterial flora has in contributing to our energy harvest and to modifying our diet and behavior. The potential importance of gut microbiota was recognized more than 1000 years ago in China where fecal transplants known as "yellow soup" were used to treat gastrointestinal disorders. Scientific community agrees that not all the bacteria are harmful and shifting the gut bacteria can be proven to be useful (Siegfried et al., 2016). Backhed et al, 2004 connected the role of microbiota in the pathogenesis of obesity and regulation of fat storage (Backhed et al., 2004). Humans have a specific bacterial flora that sets in by very young age but can be modified over the years by diet, antibiotics, xenobiotics, environment, stress and other factors that continuously change its shape and structure (Riaz Rajoka et al., 2017; Shen, 2017; Siegfried et al., 2016; Tidjani Alou, Lagier, $\&$ Raoult, 2016). Colonization of the neonatal intestine is critical for neonatal development and has a long term impact on overall health (Azad, Moossavi, Owora, \& Sepehri, 2017). Early antibiotic exposure has been associated with trends of obesity later on in life secondary to lower bacterial diversity (Azad et al., 2017). The two major gut phyla are firmicutes and bacteroidetes. The bacterial diversity of species within these groups has also been shown to be a factor for obesity. Obese animals and humans tend to have less diverse city with a higher F/B ratio. After temporary insult, eventually the microbiome could change back to initial composition (David et al., 2014). The dysbiosis in the intestine has also been blamed on increased shedding of 
114

115

116

117

118

lipopolysaccharide (LPS), an endotoxic molecule from the outer membrane of gram negative bacteria. To the contrary, microbial transfer from one individual to another increases diversity and leads to weight loss. Moreover, microbiota can produce and modify human metabolites such as bile acids that have the potential to modulate host metabolism, influence and affect behavioral and neural functions. The microbiome can also influence the immune system and the integrity of intestinal epithelium allowing endotoxins to enter the blood stream resulting in IRS along with other immune mediator disorders (Siegfried et al., 2016). An article by Zeevi et al, in nondiabetic overweight individuals (Zeevi et al.) included 800 subjects with continuously monitored week-long glucose levels of glycemic responses and microbiome changes to 46,898 meals.

Lastly, it is hypothesized that metformin exerts antio-besity effect via gut microbiome modulation in prediabetics as affects the growth of certain bacteria in microbiome composition. Metformin is widely use in prediabetes, IRS and PCOS as all are related.

\section{Fat effect}

IRS is strongly linked to fatty acid deposition with the visceral adipose tissue. Adipokines such as leptin, adiponectin, resistin, omentin, visfatin, vaspin and apelin are released from fat tissue to the general circulation, often with adverse effect. Fat storages are known major forces behind T2DM. Lipid induces adipose tissue inflammation that relates with certain defects in adipocytes (Bhattacharya \& Mukherjee, 2016). There have been conflicting studies in this area (Chowdhury et al., 2013; Hoenselaar, 2012; Siri-Tarino, Sun, Hu, \& Krauss, 2010) . For example, Siri Tarino performed a meta-analysis in 2010 of 347,747 subjects (Siri-Tarino et al., 2010). They found "no evidence for CVD risk with saturated fat “. In 2014 Chaudhry performed a meta-analysis of 512,420 subjects and discovered no associations between total saturated fatty acids and coronary risk (Chowdhury et al., 2013). In 2013, Ramsden reviewed both the Sidney 
137

138

139

140

141

142

143

144

Heart Study and the Minnesota Coronary Experiment and concluded that both had overestimated the benefits of replacing saturated fat with vegetable oils (Ramsden et al., 2013). These studies and others led Ludwig (Ludwig, 2016) to state that there had been a massive public health failure regarding the concept of saturated fat and cardiovascular disease. Nonetheless, Wang recently reported data from the NHS and HPFS studies including data with more than 3.5 million patient years. They found that replacement of saturated and trans fatty acids with unsaturated fats reduced total mortality (J. Wang et al., 2013).

\section{Other factors}

The reduction in physical activity that has occurred in our society after World War II has had a dramatic impact on insulin sensitivity of the general population. Sedentary behavior and the under use of skeletal muscle leads to impairment of glucose uptake via the GLUT4 transporter (Richter \& Hargreaves, 2013). Regarding the toxins, chemical additives have been used as flavor, color and aroma enhancers which makes certain foods irresistible and more addictive. Other toxins such as pesticides and plasticizers and the persistent exposure to them undoubtedly have an impact on hormonal regulation including insulin resistance and other metabolic disorders (Ruzzin et al., 2010).

\section{IR manifestations and diagnosis methods}

\section{Anthropometrics and physical exam}

Excess insulin has a potent proliferative effect on epidermal keratinocyte and dermal fibroblasts. These changes result in arochordons, acanthosis nigrans, androgenetic alopecia, acne and hirsutism to name a few (González-Saldivar, Ocampo-Candiani, Gómez-Flores, RodríguezGutiérrez, \& González-González, 2017). By diagnosing these manifestations timely and referring 
159

160

161

162

163

patient for further diagnosis and treatment, there can be a decrease in the obesity and diabetes burden and patients may have a better quality of life (González-Saldivar et al., 2017). The characteristic appearance of acanthosis nigrans begins with a darkening of skin at the base of the neck often seen as a ring-like presentation that surrounds the entire neck. Excess insulin has high affinity to other tyrosine kinase receptors causing epidermal growth and fibroblasts receptors. Clinical signs also include truncal obesity attributed to the insulin driven deposition of fat in the visceral adipose tissue (VAT) depot. This is best estimated by a measurement of the waist circumference (WC) and noted by increased hip to waist ratio. All fat was initially thought of simply as an energy reservoir but now is considered a metabolically active endocrine organ.

\section{Metabolic testing}

A high sensitivity C-reactive protein (hsCRP), $\mathrm{HbAlc}$ and fasting insulin levels are diagnostics factors along with nuclear magnetic resonance (NMR) lipid profile and repeated urine microalbumin. The presence of elevated CRP (synthesized in liver) has been shown to positively correlate with BMI, waist circumference and insulin resistance (Fonseca $\&$ de Oliveira Izar, 2016). NMR spectroscopy informs about the atherogenicity of lipoprotein profile thru a LPIR score (Colhoun et al., 2002). Koskinen measured LDL particle number in 1,400 healthy subjects in which, those with the highest LDL particle number were 2.8 times likelier to have metabolic syndrome than those with the lowest level of LDL particles (Koskinen et al.). The LPIR Score was developed using HOMA-IR in 4972 nondiabetic subjects and verified using glucose disposal rates measured during a hyper-insulinemic-euglycemic clamp and is significant over 50 (Shalaurova, Connelly, Garvey, \& Otvos, 2014). The homeostatic model assessment (HOMA) is a mathematical method used to quantify insulin resistance and beta-cell function with levels of $>2.4$ being considered significant, derived from a fasting plasma glucose (FPG) 
182 and fasting plasma insulin (FPI) levels. Fasting dyslipidemia and lipid panel provide useful

183 information in regard to IR and metabolic syndrome (Ray et al., 2012). Hyperglycemia alone is a

184 major factor as it is strongly related to prediabetes (Dankner, Chetrit, Shanik, Raz, \& Roth;

185 Selvin et al., 2010). Oral glucose testing (OGTT) is superior testing that needs to be incorporated 186 more as detects diabetes more efficiently than fasting glucose (FPG) as it recognizes altered post-

187 prandial metabolism. This test cannot be substituted with AIC testing as they are not equivalent 188 tests (Bartoli, Fra, \& Schianca). 


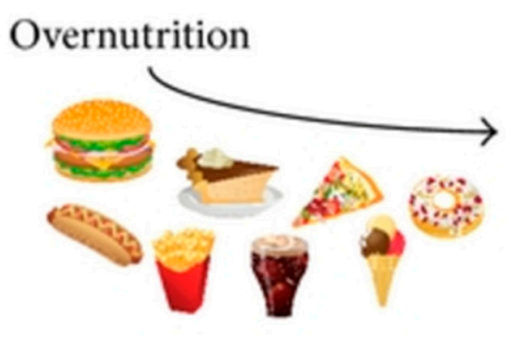

Fat accumulation

Weight gain

Hyperlipidaemia

Hyperglycaemia

Hyperinsulinaemia

Hypercholesterolaemia
Low physical activity

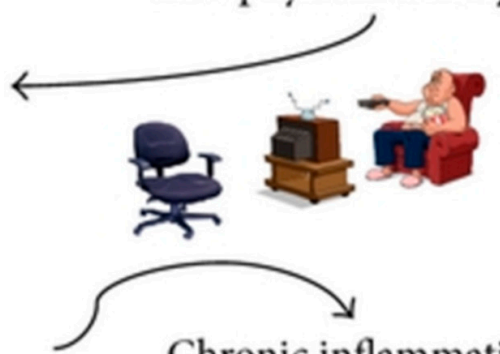

Chronic inflammation

Oxidative stress

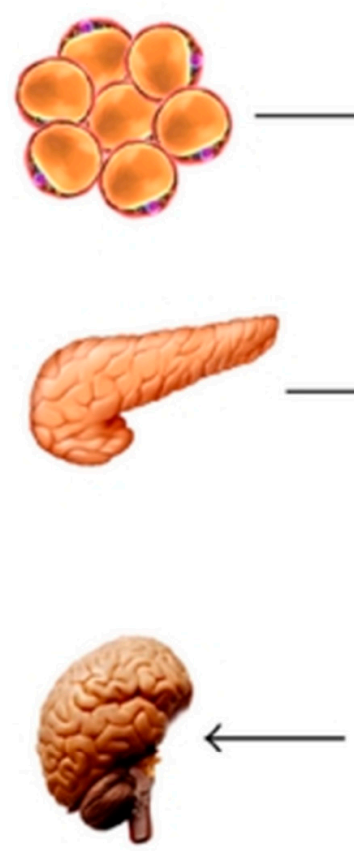

$\rightarrow$ Insulin resistance development<smiles>C[AlH]</smiles>

Type 2 diabetes development<smiles>C=[14CH2]</smiles>

$\beta$-cell failure<smiles>[CH]CC</smiles>

$\downarrow$ insulin<smiles>[CH]CC</smiles>

Impaired CNS insulin signalling and reduced brain glucose metabolism<smiles>C=IC</smiles>

Neurodegenerative disorders

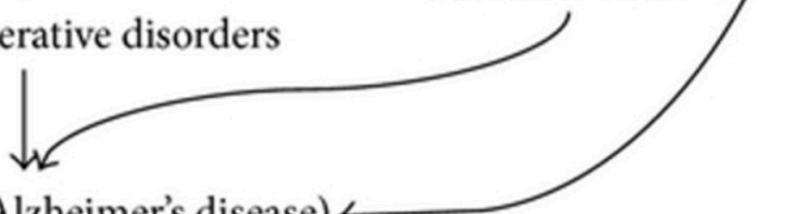

Dementia (e.g., Alzheimer's disease) 
192

193

\section{Intervening factors in Insulin resistance}

\section{Dietary modification}

The dietary goals to modify insulin resistance are moderate caloric restriction and macronutrient substitution based on the metabolic findings. The patient must achieve a dietary pattern that is sustainable over the long term and is feasible to adhere to. The purpose of achieving a sufficient caloric restriction beside losing weight is also for the health benefits as numerous studies have demonstrated that a weight loss as little as $5 \%$ has been effective in reducing IR and preventing T2DM (Tuomilehto et al., 2001). A general principle is to lower fat intake and limit carbohydrates to those of low glycemic control and maintain protein intake with respect to calorie moderation. Fiber intake also plays a factor in maintaining weight.

Furthermore, individual metabolic variations exist as well as specific genomic variations, microbiome heterogeneity, culture, geography, agriculture, etc. In 2011, Salas Salvadó et al published an analysis of food nutrients and dietary patterns for the prevention of diabetes mellitus (Salas-Salvado, Martinez-Gonzalez, Bullo, \& Ros, 2011). Foods such as sugar rich beverages, fruit juices, meats, hydrogenated oils/margarine and eggs; nutrients such as saturated fatty and trans fatty acids and dietary patterns such as high glycemic index and Western diet all increased risk of T2DM. On the other hand, foods such as whole grains, coffee and tea, low-fat milk and dairy, moderate alcohol, fruits and vegetables, nuts; nutrients such as fiber, unsaturated fatty acids, antioxidants, magnesium and dietary patterns such as low-glycemic index, Mediterranean and a low fat prudent diet all lowered the risk of T2DM. They additionally stated a low glycemic index dietary pattern that was high in fiber, magnesium and antioxidant was associated with reduced oxidation, inflammation and IRS. On the other hand, a high glycemic index dietary pattern high in saturated and trans increased oxidation, inflammation and IR. 
Weickert, 2012 looked at the concept of nutritional modulation of insulin resistance in 2012 (Weickert, 2012). He described dietary patterns in terms of insulin sensitive or insulin resistant. With similarity to Salas Salvadó, he found that poly and monounsaturates, low glycemic index, high fiber diets favored insulin sensitivity. Whereas, saturated and trans fatty acids, animal protein and high glycemic index foods favored IRS.

\section{Diet Modulation Options: Macronutrients}

\section{Choosing fats wisely}

Thru manipulation of nutrition science (Kearns, Schmidt, \& Glantz, 2016) decreasing fat and raising carbohydrates was promoted resulting in 1992 food pyramid. Lately many new food pyramids have come to light. There is little doubt that a reduction in saturated fat improves insulin sensitivity. It is fair to state that high saturated fat diets raise LDL cholesterol whereas high mono and polyunsaturated fat diets lower it which in return lowers CVD. In the Finnish Hospital study and Los Angeles study, use of dietary polyunsaturated fat lowered cholesterol significantly which translated to a reduction in cardiovascular disease (Siri-Tarino et al., 2010). Replacement of saturated fats by polyunsaturated was shown to be beneficial in a pool analysis of 11 major population cohort studies, with 344,696 participants in which saturated fat was replaced by polyunsaturates reduced heart disease. But when the saturated fats were replaced by carbohydrates, the risk of coronary disease increased (Jakobsen et al., 2009).

\section{Balancing carbohydrates}

A large number of published reports suggest that reduction of carbohydrates is the most appropriate step in modifying a diet for IR. For example, in 2002 Westman demonstrated value in 41 patients on a very low carbohydrate diet (VLCD; $<25$ g per day) (Westman, Yancy, 
237 Edman, Tomlin, \& Perkins, 2002). In 2003, Foster compared VLCD to a low-fat diet in 63

238 patients (Foster et al., 2003). Brehm, Yancy and Stern each conducted randomized clinical trials

239 showing benefit of the VLCD over a low-fat diet (Brehm, Seeley, Daniels, \& D'Alessio, 2003;

240 Stern et al., 2004; Yancy, Olsen, Guyton, Bakst, \& Westman, 2004). And most recently in 2014,

241 Bazzano, 2014 showed the benefits of a low carbohydrate diet on cardiovascular risk in 148

242 participants studied for 12 months (Bazzano et al., 2014). However, studies have shown that

243 patients regain weight due to lack of structure and lack maintenance with other studies showing

244 that weight gain may occur few years after the initial weight loss.

\section{Calories matter}

Sacks et al, 2009 compared four diets of varied carbohydrate content ( high, low

247

248

carbohydrate, high low glycemic index) and quality in 163 overweight adults (Sacks et al., 2009). After 12 weeks, no effect could be demonstrated by the influence of the carbohydrate intake. In the Pounds Lost study, Sacks, Bray and colleagues studied 811 IRS subjects. The subjects were divided into groups that consumed 4 different dietary patterns which consisted of carbohydrates, fat and protein content. At six months each dieter regardless of diet, had lost about 6 kilograms or about $7 \%$ of their body weight and eventually they regained some after 12 months. By two years, the weight loss among all groups averaged between 2.9 to 3.6 ( $p>0.20$ ). Overall regardless of diets, their insulin levels and lipid related risk factors improved (Sacks et al., 2009). 


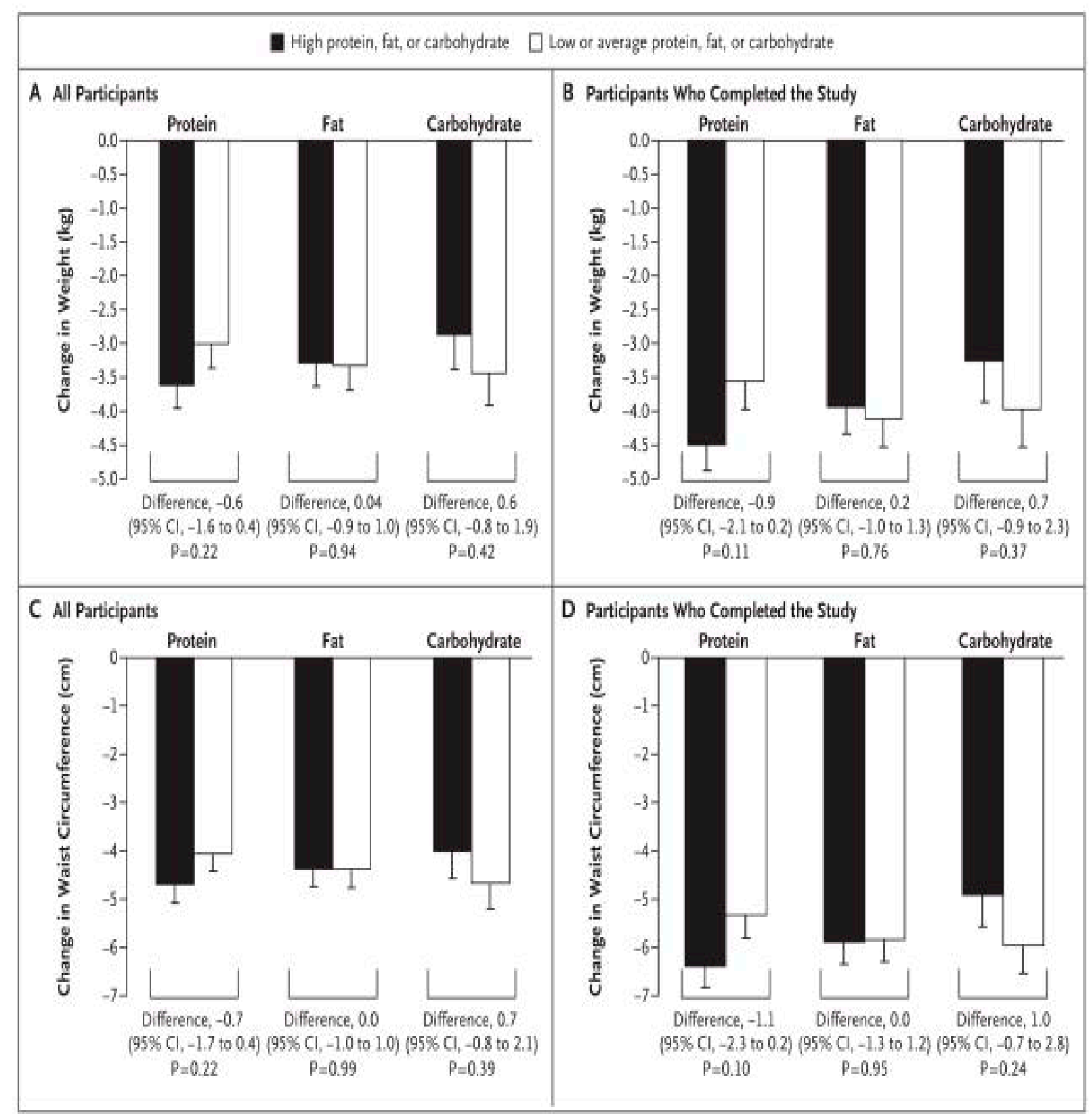

257 Fig. 2 (Sacks et al., 2009) Reproduced with permission from Massachusetts Medical Society 


\section{Maintaining protein intake}

Increase intake of protein has been promoted in the past due to the belief to have weightloss benefits. This diet change had shown to increase satiety, improve blood sugar control, reduce HOMA-IR and preserve lean body mass during weight loss (Cuenca-Sánchez, NavasCarrillo, \& Orenes-Piñero, 2015; J. O. Hill \& Peters, 2002) Ley, 2014 examined the type of protein utilized by comparing unprocessed processed and unprocessed red meat to red meat substitutes. Avoiding animal protein appears to be beneficial for IRS (Ley et al., 2014). The effect of high-protein diets on the modulation of satiety involves multiple metabolic pathways such as induction of complex signals, with peptide hormones being released from the gastrointestinal tract and blood amino acids and derived metabolites being released in the blood. Protein intake also stimulates metabolic hormones that communicate information about energy status to the brain. Long-term ingestion of high amounts of protein seems to decrease food intake, body weight, and body adiposity in many well-documented studies (Cuenca-Sánchez et al., 2015). Long-term consumption of a high-protein diet has been associated with metabolic and clinical problems, such as loss of bone mass and renal dysfunction but evidence is lacking (Cuenca-Sánchez et al., 2015).

\section{Complex Dietary Patterns in IRS}

An alternative approach to adjusting macro nutrients in the management of IR is the consideration of dietary patterns which may be beneficial. Examples of these include the DASH diet, the Healthy Eating Index/ Alternative Healthy Index diets and the Mediterranean diet. Appel and colleagues published result of the DASH diet on blood pressure in 1997 showing significant drop in arterial pressure just two weeks on the diet (Appel et al., 1997). The DASH diet reduced fasting insulin but had no effect on fasting glucose or HOMA-IR ( $p>0.8$ for the 
interaction) (Corsino et al., 2017). Adherence to the Alternative Healthy Index diet showed reversion of metabolic syndrome in individuals with central obesity and elevated triglycerides (Akbaraly et al., 2010). Significant reduction of $72 \%$ in cardiovascular events on the role of a Mediterranean diet in reducing cardiovascular events began in 1998 with the Lyon diet heart study in France. In 2004, Esposito and Giuliano conducted a 3-year trial of a Mediterranean diet in patients with metabolic syndrome in Italy (Esposito, Kastorini, Panagiotakos, \& Giugliano, 2011). They showed improvement in blood pressure, triglycerides, HDL, glucose, insulin, inflammatory markers and sexual function. They also performed in L arginine test for nitric oxide formation and found improvement after the Mediterranean diet (Esposito et al., 2011). By far the largest and most complete analysis of the Mediterranean diet was performed in Spain by the Predimed study in which over 7000 subjects were enrolled with 3287 completers studied over five years. The subjects were divided into a control group that followed an American Heart Association type low-fat diet. In Predimed, study arm one group followed a Mediterranean diet enhanced by extra-virgin olive oil while the group two subjects followed a Mediterranean diet enhanced by nuts (Estruch et al., 2013). After five years, there was a 30\% reduction in cardiovascular events. In later reports the Predimed investigators concluded that Mediterranean diets supplemented with either extra-virgin olive oil or nuts delay the onset of metabolic syndrome and are more likely to cause its reversal (Salas-Salvado et al., 2011; Sureda et al., 2016). Weichert, 2012 has reviewed dietary modification for insulin sensitivity and investigated the effects of modulating the macronutrient composition of isoenergetic diet and published comparing each intervention and its effect on weight loss, insulin resistance, diabetes risk and other effects (Weickert, 2012). 


\section{Gut Microbiome interventions}

The gut microbiota has gained attention as an active game player in development of IR, obesity and cardiovascular disease. Associated are the short chain fatty acids who contribute to the energy harvest from the foods that are eaten. The gut bacteria produce vitamin K, pantothenic acid as well water-soluble vitamins including biotin, nicotinic acid, folates, riboflavin, thiamine, pyridoxine, and cobalamin (M. J. Hill, 1997). They also produce a series of short chain fatty acids, including acetate, propionate, butyrate, TMAO and others. Short chain fatty acids (SCFA) are the primary end-products of fermentation of non-digestible carbohydrates that are absorbed by the body as fuel supplying most of the energy needs for colonocytes and approximately $10 \%$ of the total body's energy needs. But these substances also act as signals which impact lipid, glucose and insulin metabolism (Morrison \& Preston, 2016). The review from Morrison \& Preston, 2016 reveals that SCFA may have an important role during lifetime in protecting the body against deteriorating metabolic control and inflammatory status associated with Western lifestyles. Vijay-Kumar showed that microbial changes, such as antibiotic administration have a role in inducing metabolic syndrome and insulin resistance (Vijay-Kumar et al., 2010). A number of studies have shown the benefit of probiotics on reducing IR. Ruan, 2015 recently performed a meta-analysis on these reports with a total of 614 participants in which the probiotics were favored on HOMA-IR (Ruan et al., 2015). Similar results have been obtained from yogurt consumption, which can be expected to have a probiotic effect. Wang et al, 2013 reviewed Framingham cohorts including 6,526 individuals, comparing non-yogurt consumers, low intake and high intake consumers. Statistically significant improvements in triglycerides, glucose, insulin, systolic blood pressure and HOMA-IR were observed (Wang, Livingston, Fox, Meigs, \& Jacques, 2013). 


\section{Probiotics and Synbiotics}

While genetics are a nonmodifiable component of human, the microbiome could potentially be modified by lifestyle and symbiotic or probiotic supplementation to manage metabolic diseases (Barengolts, 2016). Consuming probiotics or synbiotics may improve fasting blood glucose. In a metanalysis from Nikbakht et al, 2016, the analysis of the 14 studies showed that multispecies probiotics may have a better impact than single species ones (Nikbakht et al., 2016). Dietary fiber undergoes complete or partial fermentation depending on the kind of fiber and enhances calcium absorption with the possibility to influence microbiome and bone health. Most Americans lack fiber intake. The recommended dose is 14gm/1000 calories (Wallace, Marzorati, Spence, Weaver, \& Williamson, 2017).

\section{Micronutrients}

\section{Magnesium}

Magnesium intake is also believed to be beneficial to reducing and IR. Wang, 2013 studied dietary magnesium in patients with MetS showing risk reduction in the subjects with the highest dietary magnesium intake (J. Wang et al., 2013). In addition, a meta-analysis of 13 prospective cohort studies by Dong, 2011 including 536,318 participants and 24,516 cases detected a significant inverse association between magnesium intake and risk of T2DM (relative risk (RR 0.78) (Dong, Xun, He, \& Qin, 2011). This association was regardless of geographic region, follow-up length, sex, or family history of T2DM. A significant inverse association was observed in overweight (BMI $\geq 25 \mathrm{~kg} / \mathrm{m}(2)$ ) but not in normal-weight individuals (BMI $<25$ $\mathrm{kg} / \mathrm{m}(2)$ (Dong et al., 2011). 
351

352

353

354

355

356

357

358

359

360

361

362

363

364

365

366

367

Vitamin D

An extensive literature exists about vitamin D and IRS. This includes studies on patients with normal glucose tolerance, pre-diabetes and established T2DM. Seida, 2014 performed a meta-analysis of the studies and they were unable to achieve statistical significance (Seida et al., 2014).

Omega 3 Fatty Acids

Supplementation with omega-3 fatty acids or inclusion of foods high in these essential fats has been extensively studied in IRS. The multivariable adjusted risk reduction was recently calculated through a meta-analysis performed by Kim (Kim et al., 2009). In this study the overall effect showed a modest reduction in IR with the consumption of omega-3 fatty acids.

\section{Polyphenols}

Polyphenols among others include phenolic acid, flavonoids and stilbenes. Fruit and beverages such as tea and red wine constitute the main sources of polyphenols. Certain polyphenols such as quercetin are found in all plant products (fruit, vegetables, cereals, leguminous plants, fruit juices, tea, wine, infusions, etc), whereas others are specific to particular foods (flavanones in citrus fruit, isoflavones in soya, phlorizin in apples) (Manach, Scalbert, Morand, Remesy, \& Jimenez, 2004).

Although low in polyphenols, several authors have suggested a benefit in consumption of avocado on IRS and metabolic syndrome. This was studied in detail by Fulgoni, 2013 who compared metabolic syndrome indices in avocado consumers and non-consumers (Fulgoni, Dreher, \& Davenport, 2013). They analyzed the NHANES 2001-2008 database and reviewed 17,567 subjects. Their study found statistically significant improvements in body weight, BMI, 
373 waist circumference and HDL in avocado consumers. Avocados contains good fats and slows

374 insulin release in the body and helps the person to stay full longer.

There are at least 17 clinical studies published on the benefit of green tea in metabolic syndrome and IR. Liu, 2013 performed a meta-analysis of these, including 1133 subjects (Liu et

377 al., 2013). They found a net negative change in fasting glucose, fasting insulin, HbA1c, 2-hour 378 glucose and HOMA-IR. However, in their initial analysis they were only able to demonstrate 379 statistical significance in fasting glucose, and HbA1c. The role of cinnamon has been also 380 researched for its glucose lowering properties. berries. Liu, 2014 performed a meta-analysis of seven studies, two in diabetics and five in nondiabetics. The effect was greater in diabetics, but overall HOMA-IR was reduced significantly by resveratrol (Liu, Zhou, Wang, \& Mi, 2014).

\section{Precision Medicine and recommendations}

387 physically (fig. 3) 


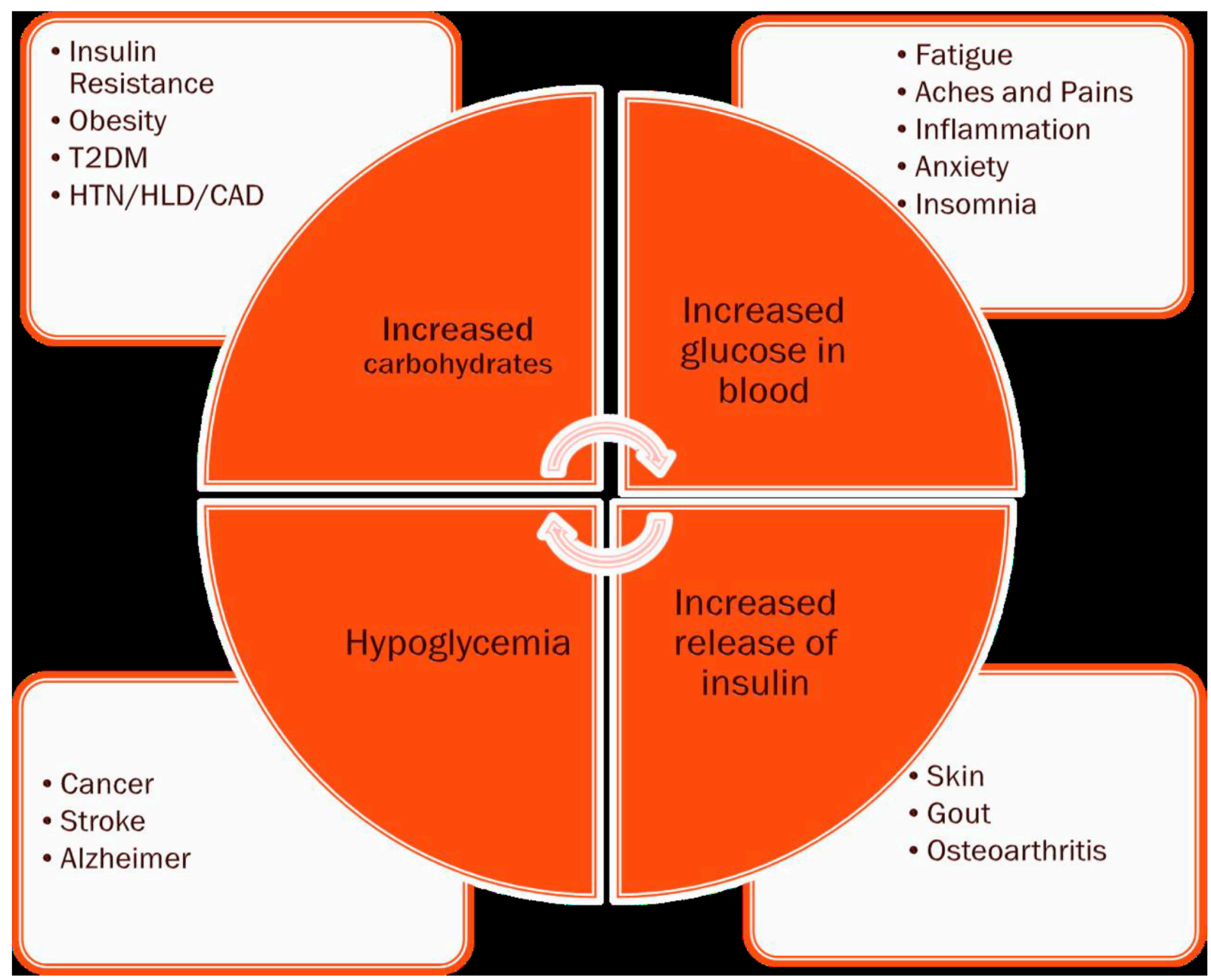

Figure 3. Insulin resistance Syndrome Cycle.

The available data reviewed in this paper points to several different approaches in the nutritional management of IRS. A large and recent literature recommends a rational approach on the macronutrient selection approach of removing saturated fats, restricting refined carbohydrates and limiting animal protein. Dietary patterns have been extensively studied as shown by the DASH, Healthy Eating Index, POUNDS LOST and Predimed studies. These inform us of the importance of dietary compliance with the largest metabolic benefit coming from moderate calorie intake and subsequent weight loss. People with IRS will benefit the most 
397

398

by combining weight loss and anti-inflammatory nutrients ( vitamins, flavonoids, and unsaturated fatty acids) (Steckhan et al., 2016).

The addition of specific foods or micronutrients may be beneficial. There is high level evidence to include monounsaturated fatty acids, probiotics (including yogurt), probiotics and synbiotics, avocado, magnesium, vitamin D, green tea and resveratrol unless contraindicated. The newly recommendation of precision/personalized medicine, taking in account the genes, environment and lifestyle, should be applied in the case of patients with IRS. While structure and accountability as well a good understanding of the condition has helped everyone, when it comes to treatment, the approach should be individualized based on what we know such a metabolism, microbiome, genetic makeup as well considering race, sex and environment. Each patient should be recommended dietary approaches based on a comprehensive assessment and diagnostic metabolic labs. One of the newly recommended biomarkers to be considered and warranted for success will be the microbiome analysis. While there is much more we don't understand yet, combating the global obesity with the knowledge and means we have will lead to preventing IRS, sensitizing insulin on the people who have IRS and lead to better outcomes and healthier lifestyles.

\section{References}

Akbaraly, T. N., Singh-Manoux, A., Tabak, A. G., Jokela, M., Virtanen, M., Ferrie, J. E., . . Kivimaki, M. (2010). Overall diet history and reversibility of the metabolic syndrome over 5 years: the Whitehall II prospective cohort study. Diabetes Care, 33(11), 2339-2341. doi:10.2337/dc09-2200

Appel, L. J., Moore, T. J., Obarzanek, E., Vollmer, W. M., Svetkey, L. P., Sacks, F. M., . . Karanja, N. (1997). A clinical trial of the effects of dietary patterns on blood pressure. DASH Collaborative Research Group. N Engl J Med, 336(16), 1117-1124. doi:10.1056/nejm199704173361601

Azad, M. B., Moossavi, S., Owora, A., \& Sepehri, S. (2017). Early-Life Antibiotic Exposure, Gut Microbiota Development, and Predisposition to Obesity. Nestle Nutr Inst Workshop Ser, 88, 67-79. doi:10.1159/000455216 
Backhed, F., Ding, H., Wang, T., Hooper, L. V., Koh, G. Y., Nagy, A., . . Gordon, J. I. (2004). The gut microbiota as an environmental factor that regulates fat storage. Proc Natl Acad Sci U S A, 101(44), 15718-15723. doi:10.1073/pnas.0407076101

Barengolts, E. (2016). GUT MICROBIOTA, PREBIOTICS, PROBIOTICS, AND SYNBIOTICS IN MANAGEMENT OF OBESITY AND PREDIABETES: REVIEW OF RANDOMIZED CONTROLLED TRIALS. Endocr Pract, 22(10), 1224-1234. doi:10.4158/ep151157.ra

Bartoli, E., Fra, G. P., \& Schianca, G. P. C. The oral glucose tolerance test (OGTT) revisited. European Journal of Internal Medicine, 22(1), 8-12. doi:10.1016/j.ejim.2010.07.008

Bays, H. E. (2011). Adiposopathy is "sick fat" a cardiovascular disease? J Am Coll Cardiol, 57(25), 24612473. doi:10.1016/j.jacc.2011.02.038

Bazzano, L. A., Hu, T., Reynolds, K., Yao, L., Bunol, C., Liu, Y., . . . He, J. (2014). Effects of lowcarbohydrate and low-fat diets: a randomized trial. Ann Intern Med, 161(5), 309-318. doi:10.7326/m14-0180

Bhattacharya, S., \& Mukherjee, S. (2016). Lipid links inflammation, immunity and insulin resistance to cause epidemic diabetes. Current Science (00113891), 110(10), 1922-1928. doi:10.18520/cs/v110/i10/1922-1928

Brehm, B. J., Seeley, R. J., Daniels, S. R., \& D'Alessio, D. A. (2003). A randomized trial comparing a very low carbohydrate diet and a calorie-restricted low fat diet on body weight and cardiovascular risk factors in healthy women. J Clin Endocrinol Metab, 88(4), 1617-1623. doi:10.1210/jc.2002021480

Chowdhury, A. M. R., Bhuiya, A., Chowdhury, M. E., Rasheed, S., Hussain, Z., \& Chen, L. C. (2013). The Bangladesh paradox: Exceptional health achievement despite economic poverty. The Lancet, 382(9906), 1734-1745. doi:10.1016/S0140-6736(13)62148-0

Chu, A. Y., Workalemahu, T., Paynter, N. P., Rose, L. M., Giulianini, F., Tanaka, T., . . . on behalf of the DietGen, C. (2013). Novel locus including FGF21 is associated with dietary macronutrient intake. Human Molecular Genetics, 22(9), 1895-1902. doi:10.1093/hmg/ddt032

Colhoun, H. M., Otvos, J. D., Rubens, M. B., Taskinen, M. R., Underwood, S. R., \& Fuller, J. H. (2002). Lipoprotein Subclasses and Particle Sizes and Their Relationship With Coronary Artery Calcification in Men and Women With and Without Type 1 Diabetes. Diabetes, 51(6), 1949-1956. doi:10.2337/diabetes.51.6.1949

Corsino, L., Sotres-Alvarez, D., Butera, N. M., Siega-Riz, A. M., Palacios, C., Pérez, C. M., . . Avilés-Santa, M. L. (2017). Association of the DASH dietary pattern with insulin resistance and diabetes in US Hispanic/Latino adults: results from the Hispanic Community Health Study/Study of Latinos (HCHS/SOL). BMJ Open Diabetes Research \&amp;amp; Care, 5(1).

Cuenca-Sánchez, M., Navas-Carrillo, D., \& Orenes-Piñero, E. (2015). Controversies Surrounding HighProtein Diet Intake: Satiating Effect and Kidney and Bone Health. Advances in Nutrition: An International Review Journal, 6(3), 260-266.

Dankner, R., Chetrit, A., Shanik, M. H., Raz, I., \& Roth, J. Basal state hyperinsulinemia in healthy normoglycemic adults heralds dysglycemia after more than two decades of follow up.

David, L. A., Maurice, C. F., Carmody, R. N., Gootenberg, D. B., Button, J. E., Wolfe, B. E., . . Turnbaugh, P. J. (2014). Diet rapidly and reproducibly alters the human gut microbiome. Nature, 505(7484), 559-563. doi:10.1038/nature12820

de la Monte, S. M., \& Wands, J. R. (2008). Alzheimer's Disease Is Type 3 Diabetes-Evidence Reviewed. Journal of diabetes science and technology (Online), 2(6), 1101-1113.

Dong, J. Y., Xun, P., He, K., \& Qin, L. Q. (2011). Magnesium intake and risk of type 2 diabetes: metaanalysis of prospective cohort studies. Diabetes Care, 34(9), 2116-2122. doi:10.2337/dc11-0518 
Esposito, K., Kastorini, C. M., Panagiotakos, D. B., \& Giugliano, D. (2011). Mediterranean diet and weight loss: meta-analysis of randomized controlled trials. Metab Syndr Relat Disord, 9(1), 1-12. doi:10.1089/met.2010.0031

Estruch, R., Ros, E., Salas-Salvado, J., Covas, M. I., Corella, D., Aros, F., . . Martinez-Gonzalez, M. A. (2013). Primary prevention of cardiovascular disease with a Mediterranean diet. N Engl J Med, 368(14), 1279-1290. doi:10.1056/NEJMoa1200303

Fonseca, F. A. H., \& de Oliveira Izar, M. C. (2016). High-Sensitivity C-Reactive Protein and Cardiovascular Disease Across Countries and Ethnicities. Clinics, 71(4), 235-242. doi:10.6061/clinics/2016(04)11

Foster, G. D., Wyatt, H. R., Hill, J. O., McGuckin, B. G., Brill, C., Mohammed, B. S., . . Klein, S. (2003). A randomized trial of a low-carbohydrate diet for obesity. N Engl J Med, 348(21), 2082-2090. doi:10.1056/NEJMoa022207

Fulgoni, V. L., 3rd, Dreher, M., \& Davenport, A. J. (2013). Avocado consumption is associated with better diet quality and nutrient intake, and lower metabolic syndrome risk in US adults: results from the National Health and Nutrition Examination Survey (NHANES) 2001-2008. Nutr J, 12, 1. doi:10.1186/1475-2891-12-1

González-Saldivar, G., Ocampo-Candiani, J., Gómez-Flores, M., Rodríguez-Gutiérrez, R., \& GonzálezGonzález, J. G. (2017). Skin Manifestations of Insulin Resistance: From a Biochemical Stance to a Clinical Diagnosis and Management. Dermatology and Therapy, 7(1), 37-51. doi:10.1007/s13555-016-0160-3

Gunay-Aygun, M., Cassidy, S. B., \& Nicholls, R. D. (1997). Prader-Willi and other syndromes associated with obesity and mental retardation. Behav Genet, 27(4), 307-324.

Heianza, Y., Ma, W., Huang, T., Wang, T., Zheng, Y., Smith, S. R., . . Qi, L. (2016). Macronutrient IntakeAssociated FGF21 Genotype Modifies Effects of Weight-Loss Diets on 2-Year Changes of Central Adiposity and Body Composition: The POUNDS Lost Trial. Diabetes Care, 39(11), 1909-1914. doi:10.2337/dc16-1111

Hill, J. O., \& Peters, J. C. (2002). Biomarkers and functional foods for obesity and diabetes. Br J Nutr, 88 Suppl 2, S213-218. doi:10.1079/bjn2002685

Hill, M. J. (1997). Intestinal flora and endogenous vitamin synthesis. Eur J Cancer Prev, 6 Suppl 1, S43-45.

Hoenselaar, R. (2012). Saturated fat and cardiovascular disease: the discrepancy between the scientific literature and dietary advice. NUTRITION, 28(2), 118-123. doi:10.1016/j.nut.2011.08.017

Jakobsen, M. U., O'Reilly, E. J., Heitmann, B. L., Pereira, M. A., Balter, K., Fraser, G. E., . . Ascherio, A. (2009). Major types of dietary fat and risk of coronary heart disease: a pooled analysis of 11 cohort studies. Am J Clin Nutr, 89(5), 1425-1432. doi:10.3945/ajcn.2008.27124

Kim, J., Lim, S.-Y., Shin, A., Sung, M.-K., Ro, J., Kang, H.-S., . . . Lee, E.-S. (2009). Fatty fish and fish omega3 fatty acid intakes decrease the breast cancer risk: a case-control study. BMC Cancer, 9, 216216. doi:10.1186/1471-2407-9-216

Koskinen, J., Magnussen, C. G., Wuertz, P., Soininen, P., Kangas, A. J., Viikari, J. S. A., . . Raitakari, O. T. Apolipoprotein B, oxidized low-density lipoprotein, and LDL particle size in predicting the incidence of metabolic syndrome: the Cardiovascular Risk in Young Finns study.

Ley, S. H., Sun, Q., Willett, W. C., Eliassen, A. H., Wu, K., Pan, A., . . Hu, F. B. (2014). Associations between red meat intake and biomarkers of inflammation and glucose metabolism in women. The American Journal of Clinical Nutrition, 99(2), 352-360. doi:10.3945/ajcn.113.075663

Liu, K., Zhou, R., Wang, B., Chen, K., Shi, L. Y., Zhu, J. D., \& Mi, M. T. (2013). Effect of green tea on glucose control and insulin sensitivity: a meta-analysis of 17 randomized controlled trials. Am J Clin Nutr, 98(2), 340-348. doi:10.3945/ajcn.112.052746

Liu, K., Zhou, R., Wang, B., \& Mi, M. T. (2014). Effect of resveratrol on glucose control and insulin sensitivity: a meta-analysis of 11 randomized controlled trials. Am J Clin Nutr, 99(6), 1510-1519. doi:10.3945/ajen.113.082024 
Lopez-Candales, A., Hernández Burgos, P. M., Hernandez-Suarez, D. F., \& Harris, D. (2017). Linking Chronic Inflammation with Cardiovascular Disease: From Normal Aging to the Metabolic Syndrome. Journal of nature and science, 3(4), e341.

Ludwig, D. S. (2016). Lowering the Bar on the Low-Fat Diet. Jama, 316(20), 2087-2088. doi:10.1001/jama.2016.15473

Manach, C., Scalbert, A., Morand, C., Remesy, C., \& Jimenez, L. (2004). Polyphenols: food sources and bioavailability. Am J Clin Nutr, 79(5), 727-747.

Morrison, D. J., \& Preston, T. (2016). Formation of short chain fatty acids by the gut microbiota and their impact on human metabolism. Gut Microbes, 7(3), 189-200. doi:10.1080/19490976.2015.1134082

Nikbakht, E., Khalesi, S., Singh, I., Williams, L. T., West, N. P., \& Colson, N. (2016). Effect of probiotics and synbiotics on blood glucose: a systematic review and meta-analysis of controlled trials. Eur $J$ Nutr. doi:10.1007/s00394-016-1300-3

Paniagua, J. A. (2016). Nutrition, insulin resistance and dysfunctional adipose tissue determine the different components of metabolic syndrome. World J Diabetes, 7(19), 483-514. doi:10.4239/wjd.v7.i19.483

Ramsden, C. E., Zamora, D., Leelarthaepin, B., Majchrzak-Hong, S. F., Faurot, K. R., Suchindran, C. M., . . Hibbeln, J. R. (2013). Use of dietary linoleic acid for secondary prevention of coronary heart disease and death: evaluation of recovered data from the Sydney Diet Heart Study and updated meta-analysis. BMJ, 346, e8707. doi:10.1136/bmj.e8707

Ray, S., Bairagi, A. K., Guha, S., Ganguly, S., Ray, D., Basu, A. K., \& Sinha, A. (2012). A simple way to identify insulin resistance in non-diabetic acute coronary syndrome patients with impaired fasting glucose. Indian J Endocrinol Metab, 16(Suppl 2), S460-464. doi:10.4103/22308210.104132

Rehman, K., \& Hamid Akash, M. S. (2016). Mechanisms of inflammatory responses and development of insulin resistance: how are they interlinked? Journal of Biomedical Science, 23, 1-18. doi:10.1186/s12929-016-0303-y

Riaz Rajoka, M. S., Shi, J., Mehwish, H. M., Zhu, J., Li, Q., Shao, D., .. Yang, H. (2017). Interaction between diet composition and gut microbiota and its impact on gastrointestinal tract health. Food Science and Human Wellness, 6, 121-130. doi:10.1016/j.fshw.2017.07.003

Richter, E. A., \& Hargreaves, M. (2013). Exercise, GLUT4, and skeletal muscle glucose uptake. Physiol Rev, 93(3), 993-1017. doi:10.1152/physrev.00038.2012

Roberts, C. K., Hevener, A. L., \& Barnard, R. J. (2013). Metabolic Syndrome and Insulin Resistance: Underlying Causes and Modification by Exercise Training. Comprehensive Physiology, 3(1), 1-58. doi:10.1002/cphy.c110062

Ruan, Y., Sun, J., Chen, R., Chen, H., He, J., \& Chen, F. (2015). Effect of probiotics on glycemic control: A systematic review and meta-analysis of randomized, controlled trials. PLOS ONE, 10(7). doi:10.1371/journal.pone.0132121

Ruzzin, J., Petersen, R., Meugnier, E., Madsen, L., Lock, E.-J., Lillefosse, H., . . Frøyland, L. (2010). Persistent Organic Pollutant Exposure Leads to Insulin Resistance Syndrome. Environmental Health Perspectives, 118(4), 465-471. doi:10.1289/ehp.0901321

Sacks, F. M., Bray, G. A., Carey, V. J., Smith, S. R., Ryan, D. H., Anton, S. D., ... Williamson, D. A. (2009). Comparison of Weight-Loss Diets with Different Compositions of Fat, Protein, and Carbohydrates. New England Journal of Medicine, 360(9), 859-873. doi:10.1056/NEJMoa0804748

Salas-Salvado, J., Martinez-Gonzalez, M. A., Bullo, M., \& Ros, E. (2011). The role of diet in the prevention of type 2 diabetes. Nutr Metab Cardiovasc Dis, 21 Suppl 2, B32-48. doi:10.1016/j.numecd.2011.03.009 
Seida, J. C., Mitri, J., Colmers, I. N., Majumdar, S. R., Davidson, M. B., Edwards, A. L., . . . Johnson, J. A. (2014). Clinical review: Effect of vitamin D3 supplementation on improving glucose homeostasis and preventing diabetes: a systematic review and meta-analysis. J Clin Endocrinol Metab, 99(10), 3551-3560. doi:10.1210/jc.2014-2136

Selvin, E., Steffes, M. W., Zhu, H., Matsushita, K., Wagenknecht, L., Pankow, J., . . Brancati, F. L. (2010). Glycated hemoglobin, diabetes, and cardiovascular risk in nondiabetic adults. The New England Journal Of Medicine, 362(9), 800-811. doi:10.1056/NEJMoa0908359

Shalaurova, I., Connelly, M. A., Garvey, W. T., \& Otvos, J. D. (2014). Lipoprotein Insulin Resistance Index: A Lipoprotein Particle-Derived Measure of Insulin Resistance. Metabolic Syndrome and Related Disorders, 12(8), 422-429. doi:10.1089/met.2014.0050

Shen, T. D. (2017). Diet and Gut Microbiota in Health and Disease. Nestle Nutr Inst Workshop Ser, 88, 117-126. doi:10.1159/000455220

Siegfried, U., Shiho, F., \& Kahn, C. R. (2016). Interactions between host genetics and gut microbiome in diabetes and metabolic syndrome. Molecular Metabolism, Vol 5, Iss 9, Pp 795-803 (2016)(9), 795. doi:10.1016/j.molmet.2016.07.004

Siitonen, N., Pulkkinen, L., Lindström, J., Kolehmainen, M., Eriksson, J. G., Venojärvi, M., ... Uusitupa, M. (2011). Association of ADIPOQ gene variants with body weight, type 2 diabetes and serum adiponectin concentrations: the Finnish Diabetes Prevention Study. BMC Medical Genetics, 12(1), 5. doi:10.1186/1471-2350-12-5

Singh, P., Somers, V. K., Romero-Corral, A., Sert-Kuniyoshi, F. H., Pusalavidyasagar, S., Davison, D. E., \& Jensen, M. D. (2012). Effects of weight gain and weight loss on regional fat distribution. American Journal of Clinical Nutrition, 96(2), 229-233. doi:10.3945/ajcn.111.033829

Siri-Tarino, P. W., Sun, Q., Hu, F. B., \& Krauss, R. M. (2010). Meta-analysis of prospective cohort studies evaluating the association of saturated fat with cardiovascular disease. Am J Clin Nutr, 91(3), 535-546. doi:10.3945/ajcn.2009.27725

Smith, D. O., \& LeRoith, D. (2004). Insulin resistance syndrome, pre-diabetes, and the prevention of type 2 diabetes mellitus. Clinical Cornerstone, 6(2), 7-13. doi:http://dx.doi.org/10.1016/S10983597(04)80050-4

Steckhan, N., Hohmann, C.-D., Kessler, C., Dobos, G., Michalsen, A., \& Cramer, H. (2016). Applied nutritional investigation: Effects of different dietary approaches on inflammatory markers in patients with metabolic syndrome: A systematic review and meta-analysis. NUTRITION, 32, 338348. doi:10.1016/j.nut.2015.09.010

Stern, L., Iqbal, N., Seshadri, P., Chicano, K. L., Daily, D. A., McGrory, J., . . . Samaha, F. F. (2004). The effects of low-carbohydrate versus conventional weight loss diets in severely obese adults: oneyear follow-up of a randomized trial. Ann Intern Med, 140(10), 778-785.

Sureda, A., Bibiloni, M. D., Martorell, M., Buil-Cosiales, P., Marti, A., Pons, A., . . Martinez-Gonzalez, M. A. (2016). Mediterranean diets supplemented with virgin olive oil and nuts enhance plasmatic antioxidant capabilities and decrease xanthine oxidase activity in people with metabolic syndrome: The PREDIMED study. Mol Nutr Food Res, 60(12), 2654-2664. doi:10.1002/mnfr.201600450

Ten, S., Bhangoo, A., Ramchandani, N., Mueller, C., Vogiatzi, M., New, M., . . Maclaren, N. (2007). Characterization of insulin resistance syndrome in children and young adults. When to screen for prediabetes? J Pediatr Endocrinol Metab, 20(9), 989-999.

Tidjani Alou, M., Lagier, J.-C., \& Raoult, D. (2016). Diet influence on the gut microbiota and dysbiosis related to nutritional disorders. Human Microbiome Journal, 1, 3-11. doi:10.1016/j.humic.2016.09.001

Tuomilehto, J., Lindström, J., Eriksson, J. G., Valle, T. T., Hämäläinen, H., Ilanne-Parikka, P., . . Uusitupa, M. (2001). Prevention of Type 2 Diabetes Mellitus by Changes in Lifestyle among Subjects with 
613

614

615

616

617

618

619

620

621

622

623

624

625

626

627

628

629

630

631

632

633

634

635

636

637

638

639

640

641

642

Impaired Glucose Tolerance. New England Journal of Medicine, 344(18), 1343-1350. doi:10.1056/NEJM200105033441801

Verdile, G., Keane, K. N., Cruzat, V. F., Medic, S., Sabale, M., Rowles, J., ... Newsholme, P. (2015). Inflammation and Oxidative Stress: The Molecular Connectivity between Insulin Resistance, Obesity, and Alzheimer's Disease. Mediators Inflamm, 2015, 105828. doi:10.1155/2015/105828

Vijay-Kumar, M., Aitken, J. D., Carvalho, F. A., Cullender, T. C., Mwangi, S., Srinivasan, S., . . Gewirtz, A. T. (2010). Metabolic syndrome and altered gut microbiota in mice lacking Toll-like receptor 5. Science, 328(5975), 228-231. doi:10.1126/science.1179721

von Holstein-Rathlou, S., BonDurant, L. D., Peltekian, L., Naber, M. C., Yin, T. C., Claflin, K. E., . . Potthoff, M. J. (2016). FGF21 Mediates Endocrine Control of Simple Sugar Intake and Sweet Taste Preference by the Liver. Cell Metab, 23(2), 335-343. doi:10.1016/j.cmet.2015.12.003

Wallace, T. C., Marzorati, M., Spence, L., Weaver, C. M., \& Williamson, P. S. (2017). New Frontiers in Fibers: Innovative and Emerging Research on the Gut Microbiome and Bone Health. J Am Coll Nutr, 36(3), 218-222. doi:10.1080/07315724.2016.1257961

Wang, H., Livingston, K. A., Fox, C. S., Meigs, J. B., \& Jacques, P. F. (2013). Yogurt consumption is associated with better diet quality and metabolic profile in American men and women. Nutrition research (New York, N.Y.), 33(1), 18-26. doi:10.1016/j.nutres.2012.11.009

Wang, J., Persuitte, G., Olendzki, B. C., Wedick, N. M., Zhang, Z., Merriam, P. A., . . Ma, Y. (2013). Dietary magnesium intake improves insulin resistance among non-diabetic individuals with metabolic syndrome participating in a dietary trial. Nutrients, 5(10), 3910-3919. doi:10.3390/nu5103910

Weickert, M. O. (2012). Nutritional modulation of insulin resistance. Scientifica (Cairo), 2012, 424780. doi:10.6064/2012/424780

Westman, E. C., Yancy, W. S., Edman, J. S., Tomlin, K. F., \& Perkins, C. E. (2002). Effect of 6-month adherence to a very low carbohydrate diet program. Am J Med, 113(1), 30-36.

Wilcox, G. (2005). Insulin and insulin resistance. Clin Biochem Rev, 26(2), 19-39.

Yancy, W. S., Jr., Olsen, M. K., Guyton, J. R., Bakst, R. P., \& Westman, E. C. (2004). A low-carbohydrate, ketogenic diet versus a low-fat diet to treat obesity and hyperlipidemia: a randomized, controlled trial. Ann Intern Med, 140(10), 769-777. 